Revue

de l'histoire des religions
Revue de l'histoire des religions

$4 \mid 2014$

L'objet rituel. Concepts et méthodes croisés

\title{
Les statues vivent aussi. Théorie néoplatonicienne de l'objet rituel
}

Statues Also Live. Neoplatonic Theory of the Ritual Object

\section{Ghislain Casas}

\section{(2) OpenEdition \\ Journals}

Édition électronique

URL : http://journals.openedition.org/rhr/8324

DOI : $10.4000 /$ rhr.8324

ISSN : 2105-2573

Éditeur

Armand Colin

Édition imprimée

Date de publication : 1 décembre 2014

Pagination : 663-679

ISSN : 0035-1423

\section{Référence électronique}

Ghislain Casas, «Les statues vivent aussi. Théorie néoplatonicienne de l'objet rituel », Revue de

l'histoire des religions [En ligne], 4 | 2014, mis en ligne le 01 décembre 2017, consulté le 19 avril 2019.

URL : http://journals.openedition.org/rhr/8324 ; DOI : 10.4000/rhr.8324 


\section{GHISLAIN CASAS}

Groupe d'Anthropologie Scolastique (GAS), Paris

Laboratoire d'étude sur les Monothéismes (LEM), Paris

\section{Les statues vivent aussi Théorie néoplatonicienne de l'objet rituel}

Comment comprendre qu'un rituel transforme une statue de pierre en un dieu vivant, et comment envisager ce phénomène autrement que comme une croyance trompeuse? Il faudrait tenter de saisir la logique paradoxale qui permet d'articuler dans un même objet ses déterminations matérielles et ses caractéristiques divines. En prenant au sérieux les analyses que les derniers philosophes néoplatoniciens ont menées de leurs propres pratiques théurgiques, il nous semble possible de dégager les linéaments d'une théorie de l'objet rituel qui remette le problème en perspective à partir de ses implications métaphysiques.

\section{Statues Also Live. Neoplatonic Theory of the Ritual Object}

How can one understand that a ritual may transform a stone statue into a living god, and see in this phenomenon something other than an erroneous belief? In order to do so, one must try to grasp the paradoxical logic that makes it possible to articulate through a single object both material determinations and divine features. By taking the analysis that the last Neoplatonists gave of their own theurgical practice seriously, it seems possible to lay the ground for a theory of the ritual object that raises the question anew, from the point of view of its metaphysical implications. 


\section{L'OBJET ET LE RITUEL}

Le court-métrage d'Alain Resnais et de Chris Marker daté de 1953, Les statues meurent aussi, apparaît comme une méditation sur ce qu'on pourrait appeler la vie et la mort des statues. Il interroge le destin des objets d'art africains dès lors qu'ils cessent d'appartenir à leur contexte religieux pour devenir des produits culturels. Le film s'ouvre sur cette assertion: «Quand les statues sont mortes, elles entrent dans l'art». C'est-à-dire: elles entrent au musée. Depuis les ready-made de Marcel Duchamp, les historiens de l'art se demandent comment un objet banal et quotidien, par exemple un urinoir, peut se transformer en œuvre d'art, par le simple fait qu'il pénètre l'espace propre du musée. Quel est ce dispositif mystérieux capable de transformer un objet en œuvre d'art par une simple opération de transfert? C'est en quelque sorte l'opération inverse que Resnais et Marker mettent en évidence dans la muséification des objets d'art africains. Si le musée offre une nouvelle vie glorieuse aux objets techniques du monde occidental, il est pour les statues africaines un véritable cimetière, le lieu de consécration de leur mort. C'est en passant du monde du culte et de la religion à celui de l'art que les statues passent de la vie à la mort.

Comment expliquer un tel passage? «Un objet est mort quand le regard vivant qui se posait sur lui a disparu». Autrement dit, les statues meurent quand elles cessent d'être objets de culte et de fixer ou de manifester la présence des esprits et des dieux et qu'elles deviennent de purs objets de contemplation. Comme on avait pour le musée sa version esthétique, on aurait là la version ethnologique du esse est percipi. Ce serait les conditions perceptuelles, au sens large, d'un objet qui en détermineraient l'essence. Ainsi le contexte du monde de l'art européen transforme-t-il une statue vivante en une statue morte, une divinité en une œuvre d'art.

L'idée paraît simple, mais comment comprendre la réciproque, qui veut que percevoir, c'est faire être ? C'est-à-dire qu'un certain usage rituel des objets les fait littéralement vivre, transforme une statue de pierre en un dieu vivant? 
Point n'est besoin pourtant d'exotisme pour rencontrer le problème du caractère vivant des objets rituels. Les religions antiques ont en effet abondamment pratiqué le rituel étonnant de l'animation des statues, que les Grecs appelaient «télestique». Si la pratique de la télestique semble appartenir à des cultes orientaux, égyptiens, chaldéens, voire iraniens, elle est largement attestée dans la culture grecque et s'est perpétuée jusque dans l'Antiquité tardive ${ }^{1}$. Elle consiste à conférer une âme à une statue en faisant descendre par des incantations et des rituels le divin dans l'objet qui le représente.

Le traité hermétique Asclépius présente cependant la télestique comme une véritable production matérielle du divin.

Ce que nous avons dit de l'homme est déjà merveilleux, mais toutes ces merveilles ne valent pas celle-ci : ce qui commande surtout l'admiration, c'est que l'homme a été rendu capable de découvrir la nature des dieux, et de la produire. Nos premiers ancêtres donc, après avoir gravement erré quant à la vraie doctrine sur les dieux - ils ne croyaient point en eux et ne se souciaient ni de culte ni de religion, - inventèrent l'art de faire des dieux; puis, l'ayant trouvé, ils y attachèrent une vertu appropriée, qu'ils tiraient de la nature matérielle; et, mêlant cette vertu à la substance des statues, comme ils ne pouvaient créer proprement des âmes, après avoir évoqué des âmes de démons ou d'anges, ils les introduisirent dans leurs idoles par des rites saints et divins, en sorte que ces idoles eussent le pouvoir de faire du bien et du mal. [...]

- Et de ces dieux qu'on nomme terrestres, ô Trismégiste, de quelle sorte est la propriété?

- Elle résulte, Asclépius, d'une composition d'herbes, de pierres et d'aromates qui contiennent en eux-mêmes une vertu occulte d'efficacité divine. Et, si l'on cherche à les réjouir par de nombreux sacrifices, des hymnes, des chants de louange, des concerts de sons très doux qui rappellent l'harmonie du ciel, c'est pour que cet élément céleste qui a été introduit dans l'idole par la pratique répétée de rites célestes

1. Sur la télestique dans le néoplatonisme, voir Joseph Bidez, «Note sur les mystères néoplatoniciens», Revue belge de philologie et d'histoire, VII, 1928, p. 1477-1481; Eric Robertson Dodds, «Theurgy and its relationship to Neoplatonism», The Journal of Roman Studies, XXXVII, 1947, p. 57-69 (repris dans The Greeks and the Irrational, Berkeley, University of California Press, 1951); Pierre Boyancé, "Théurgie et télestique néoplatoniciennes», Revue de l'histoire des religions, t. $147 \mathrm{n}^{\circ}$ 2, 1955, p. 189-209; Jean Bouffartigue, «Les statues divines du paganisme: objets artificiels ou surnaturels?», Objets sacrés, objets magiques de l'Antiquité au Moyen-âge, éd. Charles Delattre, Themam, CNRS, 2007, p. 53-64; Sarah Iles Johnston, «Animating Statues: A Case Study in Ritual», Arethusa, 41.3, 2008, p. 445-478; Jan N. Bremmer, «The Agency of Greek and Roman Statues: from Homer to Constantine», Opuscula, Annual of the Swedish Institutes at Athens and Rome, 6, 2013, p. 7-21. 
puisse supporter joyeusement ce long séjour parmi les hommes. Voilà comment l'homme fabrique des dieux ${ }^{2}$.

Si la pratique consiste littéralement à joindre une âme à une composition matérielle particulière, elle est interprétée comme une fabrication humaine du divin: homo est fictor deorum ${ }^{3}$. L'homme est un fabricant de dieux. Que signifie faire un dieu? Est-ce simplement fabriquer une idole matérielle, une statue qui n'est que le réceptacle d'une puissance supérieure? Ou bien en va-t-il d'une production d'ordre supérieur, pour ainsi dire ontologique, dans laquelle l'homme véritablement ferait les dieux?

Le problème est exposé sur un mode parodique chez Minucius Felix, qui, sous couvert de tourner en ridicule la télestique païenne et son caractère idolâtre, en expose toute l'ambiguïté et toute la subtilité :

Qui peut donc douter que, si la foule adresse des prières et rend un culte public aux effigies consacrées de ces personnages, c'est parce que l'opinion, l'esprit des gens ignorants se laisse abuser par les grâces de l'art, éblouir par l'éclat de l'or, fasciner par le brillant de l'argent et la blancheur de l'ivoire? Quiconque se représentera les instruments de torture et les machines qui œuvrent à façonner toute statue, rougira de craindre une matière dont s'est joué l'artiste pour en faire un dieu. En effet, un dieu de bois, qui peut être un fragment de bûches ou de cruche stérile, est suspendu, taillé, dégrossi, raboté; un dieu de bronze ou d'argent provient bien souvent, comme cela fut le cas pour un roi d'Égypte, d'un immonde petit récipient, qui est fondu, battu à coup de maillet et façonné sur l'enclume; un dieu de pierre est taillé, sculpté et poli par un homme corrompu, et d'ailleurs hoffmann eros aletheia pistisil est aussi insensible aux outrages de sa naissance que plus tard aux honneurs dont l'entoure votre vénération. On me dira peut-être que la pierre, le bois ou l'argent n'est pas encore un dieu. Quand donc celui-ci naît-il ? Voyez-le couler, forger, sculpter: il n'est pas encore un dieu; voyez-le souder, assembler, ériger: il n'est pas encore un dieu; voyez-le parer, consacrer, implorer: alors enfin il est dieu, lorsqu'un homme l'a voulu tel et décidé comme tel ${ }^{4}$.

Le texte semble distinguer trois moments de la production de la statue divine: la fabrication, l'érection et la consécration; mais

2. Asclépius, 37-38, Corpus Hermeticum, t. II, éd. A.D. Nock, trad. AndréJean Festugière, Paris, Les Belles Lettres, 1946, p. 347-349.

3. Sur cette célèbre formule, voir Sarah Iles Johnston, «Homo fictor deorum est: Envisionning the Divine in Late Antique Divinatory Spells», in J. Bremmer and A. Erskine (eds.), The Gods of Ancient Greece, Edinburgh University Press, 2010, p. 406-21.

4. Minucius Felix, Octavius, XXIV, 8, éd. et trad. Jean Beaujeu, Paris, Les Belles Lettres, 1964, p. 38-39. 
ce n'est qu'avec le dernier que la statue devient véritablement un dieu. Une opposition claire apparaît entre la production matérielle et la production rituelle. Ce qu'on peut appeler à proprement parler fabrication du divin, ce n'est pas la production de la statue comme objet matériel, mais sa production symbolique et rituelle, qu'effectuent la parure et la prière. C'est performativement, pour ainsi dire, que la statue devient un dieu.

Est-il possible de lire à rebrousse-poil le texte de Minucius Felix et de tenter de prendre au sérieux la distinction qu'il propose? En s'en tenant à une lecture faible, on opposera la production matérielle de l'objet, la fabrication de la statue concrète, à la projection symbolique et performative sur elle de qualités divines, qui relève d'un phénomène de croyance. Si l'on accepte en revanche de donner un sens fort à la distinction, ce qui vient compléter la fabrication matérielle de l'objet n'est pas un supplément d'âme fictif mais un autre type de production qui, s'il ne fabrique rien à proprement parler, fait du divin. Il ne s'agira plus alors d'opposer, comme le fait Minucius Felix, une réalité objective et sa déformation par une perception subjective, la statue et ce qu'on croit qu'elle est, mais plutôt d'articuler deux niveaux d'objectivité, si l'on veut, matérielle et spirituelle, qui font exister la statue non seulement comme objet mais aussi comme dieu.

Nous voudrions tenter de comprendre en quel sens l'activité rituelle qui entoure l'objet peut être considérée comme une activité productive. Dans le rite de consécration de la statue s'opère un basculement de point de vue: la statue qui n'était qu'un tas de pierre devient une divinité vivante. Nous faisons l'hypothèse que ce basculement entre les deux perspectives ne doit pas être envisagé comme une simple différence subjective de point de vue, comme telle externe à l'objet, mais au contraire comme un court-circuit interne à l'objet lui-même, qui le scinde objectivement. L'idée que la distinction dépendrait du point de vue de celui qui perçoit l'objet n'est pas satisfaisante: si l'on s'accorde aisément à dire que prêter une âme ou une qualité divine à une chose est une vue de l'esprit, accepte-t-on de dire que considérer une chose comme un pur agrégat de matière en est une aussi? Il nous paraît plus éclairant de comprendre comment un objet peut se présenter aussi bien comme matière que comme esprit.

Ce qui est difficile à saisir, c'est qu'un même objet soit à la fois un tas de pierre et un dieu vivant, que se superposent en lui ces deux 
dimensions qui en font l'unité profonde en même temps qu'elles le séparent de lui-même, le partagent entre ce qu'il est matériellement et substantiellement et ce qu'il est en tant que puissance divine. Il nous semble pourtant que seule une telle objectivité paradoxale permet de comprendre la télestique. En même temps, ce n'est que dans la pratique rituelle qu'une telle objectivité existe et se vérifie. Ce n'est en effet que par le moyen du rite que la statue devient un dieu. Il importe alors de comprendre en quel sens le rituel permet d'articuler les différentes dimensions de l'objet afin, selon la formule hermétique, de faire des dieux.

Nous appellerons objet rituel l'objet qui est au centre du rituel, à la fois en tant que ce qui fait l'objet du culte (la statue que l'on vénère) et ce que le culte produit (la statue transformée en dieu). Nous voudrions mettre en évidence dans le syntagme objet rituel la relation circulaire qui existe entre l'objet et le rituel, au sens où si c'est une détermination de l'objet lui-même qui rend possible le rituel, ce n'est que dans le rituel que cette détermination se déploie.

\section{MÉTAPHYSiQue de L'OBJet RITUEL}

Nous nous attacherons au cas des pratiques théurgiques et des développements théoriques qu'elles ont suscité dans le dernier néoplatonisme à partir du $\mathrm{IV}^{\mathrm{e}}$ siècle $\mathrm{e}^{5}$ Le contraste entre le développement d'une philosophie extrêmement abstraite et subtile et l'attrait pour des formes archaïsantes et superstitieuses de paganisme, qui a longtemps étonné les historiens de la pensée, révèle ici toute sa force. Comment les raffinements métaphysiques du néoplatonisme ont-ils pu s'accommoder d'une pratique religieuse aussi grossière que celle de l'animation des statues? Loin qu'il y ait une contradiction chez les néoplatoniciens entre

5. Pour une présentation générale de la théurgie, la référence reste l'ouvrage classique de Hans Lewy, Chaldean Oracles and Theurgy, Mysticism, Magic and Platonism in the Later Roman Empire, nouvelle éd. Michel Tardieu, Paris, Brepols, «Études Augustiniennes, 77», 1978; au sujet de la théurgie dans les milieux néoplatoniciens, voir l'étude de Carine Van Liefferinge, La Théurgie, des Oracles Chaldaïques à Proclus, Liège, Centre International d'Étude de la Religion Grecque Antique, «Kernos, Suppl. 9», 1999. Pour une tentative de relativisation des sources néoplatoniciennes, voir Ilinca Tanaseanu, Theurgy in Late Antiquity: The Invention of a Ritual Tradition, Göttingen: Vandenhoech \& Ruprecht, 2013. 
la métaphysique et les usages religieux, il existe plutôt entre elles une affinité profonde, si bien que le néoplatonisme offre l'une des explications théoriques les plus élaborées et les plus pénétrantes des rituels télestiques.

Plotin déjà semble avoir reconnu les pratiques télestiques comme une donnée culturelle irréfutable, sans toutefois leur accorder une place trop importante dans sa pensée.

Les anciens sages qui ont voulu se rendre les dieux présents en construisant des temples et des statues, me paraissent avoir bien vu la nature de l'univers; ils ont compris qu'il est toujours facile d'attirer l'âme universelle, mais qu'il est particulièrement aisé de la retenir, en construisant un objet disposé à subir son influence et à en recevoir la participation. Or la représentation imagée d'une chose est toujours disposée à subir l'influence de son modèle, elle est comme un miroir capable d'en saisir l'apparence. La nature, avec un art admirable, fait les choses à l'image des êtres dont elle possède les raisons; ainsi est née chaque chose, raison intérieure à la matière, recevant une forme correspondante à une raison supérieure à la matière; car la nature la met en contact avec la divinité d'après laquelle elle est engendrée, que l'âme universelle contemple, et d'après laquelle elle se dispose en créant la chose. Il est donc impossible qu'il n'y ait rien qui ne participe à cette divinité; mais il est aussi impossible qu'elle descende ici-bas ${ }^{6}$.

Pour Plotin, la magie pratique n'est qu'un cas des lois générales de sympathie qui gouvernent l'Univers. Si la magie est opérante, c'est parce que le monde est animé de part en part et que toutes ses parties communiquent et conspirent. La première magie, c'est la nature elle-même. En vertu de cette sympathie universelle, une parcelle de matière peut recevoir l'influence des dieux.

Le rapport entre la statue et la divinité est celui d'une image à son modèle. S'il y a de toute évidence dans la relation de ressemblance bien plus qu'une simple analogie formelle, une sorte de connivence et de continuité entre les choses qui se ressemblent, on ne saurait toutefois en faire un vecteur de présence. Les choses qui se ressemblent ne se confondent pas et Plotin prend soin de préciser que la divinité ne descend pas dans la matière.

Le contre-exemple plotinien permet de saisir l'ampleur du tournant effectué par Jamblique, qui, loin de se réduire à la simple adoption, sous l'influence des Oracles chaldaïques, de pratiques

6. Plotin, Énnéades, IV, 3 [27], 11: «Difficultés relatives à l'âme I», trad. Émile Bréhier, Paris, Les Belles Lettres, 1927, p 78. 
magico-religieuses orientales, consiste en une véritable révolution métaphysique.

En effet, comme il fallait aussi que ce qui est sur terre ne fût nullement sans part à la communauté divine, la terre aussi a reçu une certaine part divine de celle-ci, capable d'offrir un espace pour accueillir les dieux. Cela, assurément, l'art théurgique l'a bien vu et ainsi découvre, selon le principe général de l'appropriation, les réceptacles qui conviennent à chacun des dieux: aussi entrelace-t-il souvent pierres, herbes, êtres vivants, aromates et autres choses de ce genre, sacrées, achevées et spécifiquement divines, et ensuite réalise à partir de tout cela un réceptacle complètement achevé et pur.

Car il ne faut pas rejeter toute la matière, mais seulement celle qui est étrangère aux dieux, il faut plutôt choisir celle qui leur est appropriée, dans la pensée qu'elle est capable de s'harmoniser à la construction de leurs demeures, à l'érection de leurs statues et à l'accomplissement sacré des sacrifices. Car, s'il en était autrement, pour les dieux terrestres ou les hommes qui habitent ici, il ne pourrait y avoir de participation à la réception des êtres supérieurs, si un tel fondement n'avait pas été préalablement établi. Il faut suivre les paroles secrètes qui disent que, par les spectacles bienheureux, une certaine matière est également offerte, venant des dieux. Celle-ci est d'une certaine manière connaturelle à ceux-là mêmes qui la donnent. Aussi, le sacrifice d'une matière éveille les dieux pour qu'ils se manifestent, les appelle aussitôt à descendre pour se laisser saisir, leur offre un espace de réception quand ils se présentent, et les montre parfaitement ${ }^{7}$.

La confection des statues et leur animation ne sont pas de simples traits culturels empruntés à la religion des Chaldéens, mais le corrélat pratique d'une nouvelle conception de la matière ${ }^{8}$. Sous certaines conditions, la matière est connaturelle aux dieux, et à la limite, elle est elle-même divine. La théurgie est une véritable opération métaphysique: en faisant descendre les dieux dans les statues, elle réveille dans la matière ses dispositions au divin, elle réunit le cosmos entier. Si la religion professée par Jamblique consiste en une série d'activités matérielles, c'est parce que les dieux sont aussi dans la matière et que, si le théurge désire s'unir à

7. Jamblique, Les mystères d'Égypte, Réponse d'Abamon à la Lettre de Porphyre à Anébon, trad. Michèle Broze et Carine Van Liefferinge, Bruxelles, Ousia, 2009, p. 136-137. Nous ne pouvons que signaler la nouvelle édition et traduction du texte, parue entre-temps, aux éditions de Belles Lettres, Réponse à Porphyre, éd. et trad. H.-D. Saffrey et A.-Ph. Segonds, avec la collaboration de A. Lecerf, Paris, Les Belles Lettres, 2013.

8. Pour une étude détaillée de la place de la théurgie dans la philosophie de Jamblique, voir Gregory Shaw, Theurgy and the Soul: the neoplatonism of Iamblichus, University Park, Pennsylvania State University Press, 1995. 
eux, il ne peut le faire sans prendre celle-ci en considération. Sans récuser le caractère transcendant du divin, Jamblique n'en jette pas moins, en faisant l'apologie de la théurgie, les bases de ce que nous nous risquerons à appeler une théologie matérialiste.

C'est dans le fragment Sur l'art hiératique de Proclus que cette intuition a été poussée le plus loin. Dans un texte en apparence simple, qui s'apparente à un petit traité de magie pratique, Proclus livre une théorie de l'objet rituel qui présuppose largement la métaphysique qu'il développe dans ses ouvrages systématiques.

De même que les dialecticiens de l'amour s'élèvent à partir des beautés sensibles jusqu'à ce qu'ils rencontrent le principe même unique de toute beauté et de tout intelligible, ainsi les initiateurs aux saints mystères, partant de la sympathie qui unit toutes les choses visibles entre elles et avec les puissances invisibles, et comprenant que tout est en tout, ont-ils fondé cette science hiératique, non sans s'émerveiller de voir dans les premiers termes des chaînes les termes les plus infimes et dans ces derniers les tout premiers, au ciel les choses terrestres dans leur cause et sous un mode céleste, ici-bas les choses célestes sous un mode propre à la terre. D'où vient en effet que l'héliotrope se meut en accord avec le Soleil, le sélénotrope avec la Lune, tous deux faisant cortège, dans la mesure de leurs forces, aux luminaires du Monde ? ${ }^{9}$

Le texte se présente comme une réflexion sur le thème tout à fait classique en magie de la «sympathie universelle». La confection de mélanges de différentes matières servant à remplir les statues creuses pour y loger des dieux, repose tout entière sur les propriétés magiques ou sympathiques des animaux, des végétaux et des minéraux. C'est parce que la matière et le divin, la terre et le ciel sont en sympathie qu'une activité magique est possible. Il ne s'agit toutefois pas d'utiliser les propriétés naturelles des choses, pas plus qu'il n'est question de tirer parti de leurs vertus surnaturelles ou occultes. Proclus entend montrer que, selon le mot de Thalès, «tout est plein de dieux $\gg^{10}$ et ce qui précisément l'intéresse dans les propriétés divines des choses, c'est qu'elles témoignent du mode de présence des dieux dans les choses, qu'elles en sont le signe secret.

Tout est donc plein de dieux, la terre est pleine de dieux célestes, le ciel de dieux supracélestes; chaque série procède, s'accroissant en nombre, jusqu'à ses termes derniers. En effet, ce qui existait dans l'unité avant toutes choses est manifesté dans tous les membres de

9. Proclus, Sur l'art hiératique, trad. A.-J. Festugière, in La révélation d'Hermès Trismégiste, vol. 1, Paris, Les Belles Lettres, 2006 (1944), p. 134.

10. Fr. A 22 D-K, voir Aristote, De l'âme, I, 5, 411a 5-10. 
la série. D'où les organisations des âmes, dépendantes celles-ci d'un dieu, celles-là d'un autre ${ }^{11}$.

Les puissances divines sont fragmentées et réparties dans les choses. Il y a au cœur de chaque chose, confondue avec son intimité ou son individualité la plus propre, une étincelle divine. Cet indice qui ordonne la chose à un dieu, les néoplatoniciens lui donnent le nom technique de symbole (sumbolon) ou de signature (sunthêma).

Car, celui qui est cause de tout l'univers a ensemencé dans tous les êtres des marques de son absolue supériorité; par le moyen de ces marques, il a établi tous les êtres en référence à lui, et il est ineffablement présent à tous, bien qu'il transcende tout l'univers. Donc chaque être, en rentrant dans ce qu'il y a d'ineffable dans sa propre nature, découvre le symbole du Père de tout l'univers; tous les êtres par nature le vénèrent et, par le moyen de la marque mystique qui appartient à chacun, s'unissent à lui, en dépouillant leur propre nature et en mettant tout leur cœur à ne plus être que la marque de dieu et ne plus participer que de dieu, à cause du désir qu'ils ont de cette nature inconnaissable et de la Source du Bien $[\ldots]^{12}$.

La signature est l'indice d'une présence divine, non pas vecteur de signification mais d'efficacité13. C'est un symbole efficace, qui agit dans l'objet, le convertit au dieu qui le constitue. Il ne s'agit pas pour le théurge de l'interpréter mais d'opérer avec, de le faire agir et d'en libérer la puissance. La théurgie reconduit les

11. Ibid., p. 135.

12. Proclus, Théologie platonicienne, II, 8, éd. et trad. Henri-Dominique Saffrey et Leendert Gerrit Westerink, Paris, Les Belles Lettres, 1974, p. 56.

13. Traditionnellement la théorie des signatures se situe au croisement de la botanique, de la médecine, de l'alchimie et de la magie, et procède de l'idée que la ressemblance ou la similitude entre les choses permet leur action les unes sur les autres, en particulier en ce qui concerne le rapport entre la morphologie des plantes et leurs vertus curatives. Voir D. Ball-Simon et P. Daszkiewicz, L'héritage oublié des signes de la nature, La loi des signatures, Paris, Les deux océans, 1999. La tradition médicale qui va de Théophraste à Paracelse donne lieu, chez Jacob Boehme, à une théorie générale de la création, cf. De la signature des choses, trad. P. Deghaye, Paris, Grasset, 1995. Concernant le mode de signification particulier de la signature, en rapport avec les notions de ressemblance et d'efficacité, voir Michel Foucault, Les mots et les choses, ch. II, II «Les signatures », Paris, Éditions Gallimard, 1966, p. 40-45 et Giorgio Agamben, «Théorie des signatures», Signatura rerum. Sur la méthode, trad. Joël Gayraud, Librairie philosophique J. Vrin, Paris, 2008, p. 37-91. Nous traduisons sunthèma par «signature» en raison de la proximité entre la doctrine néoplatonicienne de la théurgie et la théorie médicale et magique des signatures, dans lesquelles les signes visibles du monde renvoient aux puissances invisibles avec lesquelles ils permettent d'opérer, comme de véritables «mots de passe» institués par les dieux. 
choses à leur divinité. Cela suppose qu'elles se dépouillent de leurs déterminations naturelles: la signature est précisément la marque dans l'objet de la séparation entre sa nature et sa divinité.

D'où encore, par exemple, le grand nombre des animaux héliaques, tels que le lion et le coq, qui eux aussi participent au divin selon le rang qu'ils occupent. L'admirable, c'est comment, chez ces animaux, les moins pourvus en force et en taille se font craindre de ceux qui l'emportent sur ces deux points: car le lion, dit-on, recule devant le coq. La raison n'en est pas à prendre dans les données des sens, mais dans une considération intellectuelle, c'est-à-dire une différence qui remonte aux causes elles-mêmes. C'est que, en vérité, la présence dans le coq des symboles héliaques a plus d'efficace. Il le montre bien par la conscience qu'il a du circuit du Soleil: car il chante un hymne au lever de l'astre et quand l'astre se tourne vers les autres centres... ${ }^{14}$

Si le lion est naturellement plus fort que le coq, toutefois la signature solaire est plus puissante dans le coq que dans le lion. C'est pourquoi le coq peut l'emporter sur le lion. Parallèlement à l'ordre naturel des choses, il existe un ordonnancement divin du monde, qui ne se superpose pas exactement à celui-là ${ }^{15}$. Si les êtres se distinguent les uns des autres par des caractéristiques formelles générales, ils se rapportent aussi les uns aux autres en fonction de propriétés divines plus particulières. Ils appartiennent à la fois à des classes ontologiques et à des ordres divins. La signature est précisément ce qui soustrait l'objet à ses déterminations naturelles ou essentielles, et l'ordonne à un mode d'existence proprement divin.

Quel est ce mode d'existence non naturel et pour ainsi dire non ontologique des choses? On peut l'appeler symbolique, au sens précis du symbole dans la théurgie néoplatonicienne, ou encore rituel. Le rituel ne consiste pas simplement en une technique ou une activité humaine mais c'est une dimension des choses.

Le lotus lui aussi manifeste son affinité avec le Soleil: sa fleur est close avant l'apparition des rayons solaires, elle s'ouvre doucement

14. Proclus, Sur l'art hiératique, p. 135.

15. C'est, nous semble-t-il, la signification profonde de la théorie proclienne des hénades. Leur fonction ne se limite pas à combler l'écart entre l'un et l'être, mais donne à penser une causalité qui n'est pas celle des formes intelligibles. Bien que Proclus ne soit jamais tout à fait explicite à ce propos, il y a une différence fondamentale entre l'hénadologie et l'ontologie, dont les commentateurs n'ont pas suffisamment rendu compte. À ce sujet on se reportera aux remarques de Radek Chlup, Proclus. An introduction, Cambridge, Cambridge University Press, 2012, en particulier p. 112-136, ainsi qu'à la thèse de Edward P. Butler, The Metaphysics of Polytheism in Proclus, New School University, avril 2003. 
quand le Soleil commence à se lever, et à mesure que l'astre monte au zénith, elle se déploie, puis de nouveau se replie lorsqu'il s'abaisse vers le couchant. Or quelle différence y a-t-il entre le mode humain de chanter le Soleil, en ouvrant ou fermant la bouche et les lèvres, et celui du lotus, qui déplie et replie ses pétales ? Car ce sont là ses lèvres à lui, c'est là son chant naturel.

Mais pourquoi parler des plantes, où subsiste encore quelque trace de vie générative? Ne voit-on pas les pierres elles-mêmes respirer en correspondance avec les effluves des astres? Ainsi l'hélite, par ses rayons à couleur d'or, imite les rayons du Soleil; la pierre qu'on nomme «œil de Bel» et dont l'aspect ressemble aux prunelles des yeux émet du centre de sa prunelle une lumière brillante, ce qui fait dire qu'on devrait l'appeler «œil du Soleil»; la sélénite change de forme et de mouvement en accord avec les changements de la Lune, et l'héliosélène est comme une image de la rencontre des deux luminaires, à la ressemblance des rencontres et des séparations qui se font au ciel ${ }^{16}$.

S'il est possible d'invoquer une divinité, de la faire descendre dans une statue, c'est parce que la statue elle-même œuvre à faire venir en elle le divin. Pour le dire autrement, l'activité rituelle des hommes ne fait que doubler l'existence rituelle des choses. Toute chose a une vie rituelle propre : les plantes et les pierres prient à leur façon. En décrivant le monde comme une grande prière cosmique, Proclus semble décliner la très belle théorie plotinienne de la contemplation.

Avant d'aborder notre sujet sérieusement, si nous nous amusions à dire que tous les êtres désirent contempler et visent à cette fin, les êtres raisonnables comme les bêtes, et même les plantes et la terre qui les engendre; si nous ajoutions que tous ces êtres arrivent à cette fin autant qu'ils en sont capables et conformément à leur nature, mais qu'ils contemplent chacun à leur manière et atteignent tantôt des réalités, pourrait-on supporter pareil paradoxe ? [...] Sans doute ces vies sont des pensées de différentes espèces; il y a une pensée de la plante, une pensée de l'animal doué de sens, une pensée de l'être doué d'une âme; comment des pensées? Oui, puisque ce sont des raisons; toute vie est une pensée, mais une pensée plus ou moins obscure comme la vie elle-même ${ }^{17}$.

De même que selon Plotin, la vraie vie est une contemplation et qu'il n'y a qu'une différence de degré entre l'activité contemplative de l'esprit pur et celle des plantes et des pierres, on pourrait dire avec Proclus que la vie est fondamentalement rituelle, à des degrés divers.

16. Proclus, Sur l'art hiératique, p. 134-135.

17. Plotin, Énnéades, III, 8 [30], 8: «De la nature, de la contemplation et de l’Un», trad. É. Bréhier, Paris, Les Belles Lettres, 1999, p. 277-279. 
Car tous les êtres prient selon le rang qu'ils occupent, ils chantent les chefs qui président à leur série tout entière, chacun louant à sa manière, spirituelle, rationnelle, physique ou sensible: ainsi l'héliotrope se meut-il autant qu'il lui est facile de se mouvoir, et si l'on pouvait entendre comme il frappe l'air durant qu'il tourne sur sa tige, on se rendrait compte à ce bruit qu'il offre une sorte d'hymne au Roi, tel qu'une plante peut le chanter ${ }^{18}$.

Le rituel est le processus par lequel chaque chose se convertit vers le principe divin dont elle procède et fait retour vers lui, comme la fleur héliotrope se tournant vers le soleil. L'existence est pensée dans le néoplatonisme comme un processus dynamique, comme un jeu entre l'origine et le retour. C'est ce qu'énonce la proposition 35 des Éléments de théologie: «Tout ce qui est causé demeure dans sa propre cause, procède d'elle et se convertit vers elle $»^{19}$. En appliquant le modèle de la manence (monè), de la procession (proodos) et de la conversion (epistrophè) à notre propos, on pourrait dire de façon schématique que les déterminations naturelles d'une chose sont ce par quoi elle se sépare de sa cause divine, la signature qu'elle porte en elle la marque de l'immanence du divin en elle et son activité rituelle sa façon de faire retour vers son origine divine ${ }^{20}$.

Une autre signification de l'activité rituelle humaine semble se dégager de ces considérations. Ce que vise la théurgie, ce n'est pas un usage magique des propriétés des choses mais ce n'est pas non plus une projection sur les choses d'un imaginaire religieux. Pour échapper à cette alternative, le coup de force de Proclus est de dire que l'activité symbolique est immanente aux choses. Les choses ne sont pas des symboles des dieux: elles sont divines par les symboles qui agissent en elles. La vie symbolique ne relève pas de l'anthropologie mais de la métaphysique. Les pratiques rituelles n'ajoutent pas une dimension symbolique aux choses mais visent

18. Proclus, Sur l'art hiératique, p. 134.

19. Proclus, The Elements of Theology, éd. E.R. Dodds, Oxford, Clarendon Press, 1963, p. 38 (nous traduisons).

20. Il faudrait nuancer cette interprétation, en relisant par exemple la proposition 39 des Éléments de théologie, dans laquelle il apparaît clairement que la conversion peut s'effectuer selon l'être, la vie ou la connaissance, c'est-à-dire selon la nature propre des différentes classes d'êtres. La question est au fond de savoir si l'hénadologie ne permettrait pas, dans une certaine mesure, de courtcircuiter l'ontologie et de mettre en évidence un léger décalage entre les différentes causalités à l'œuvre dans la procession et la conversion des êtres. 
au contraire à correspondre avec l'activité rituelle des choses ellesmêmes et à l'amplifier. C'est en ce sens qu'on peut dire que les choses se réalisent pleinement dans la pratique rituelle des hommes, qu'elles touchent plus parfaitement à leur divinité, et que donc les hommes «font des dieux». L'activité rituelle est productive dans la mesure où elle est une démiurgie symbolique: en elle, les objets connaissent une deuxième genèse, qui n'est plus de l'ordre d'une production substantielle mais d'une reproduction symbolique. Le rituel théurgique, en actualisant la puissance symbolique des choses, en activant les signatures divines qui logent en leur cœur, produit les objets en tant que divins en les reproduisant symboliquement. Ce qui est en jeu dans la théurgie, c'est une réévocation de l'ordre du monde sous l'aspect de sa divinité.

\section{L'EFFICACITÉ SANS LA CROYANCE}

Nous souhaiterions faire le point, pour conclure, sur les difficultés que présente l'adoption d'un tel modèle en vue d'une analyse de la fonction de l'objet rituel, en particulier dans l'interprétation des pratiques télestiques.

La première difficulté tient au statut du discours néoplatonicien lui-même. Le fait que les mêmes personnes qui ont pratiqué la théurgie l'aient aussi pensée pose sans aucun doute un problème épistémologique majeur. Comment accepter comme valide une explication qui est de l'ordre d'une théologie, voire d'une apologie? Peut-on prendre au sérieux les explications d'un phénomène religieux données par ceux-là mêmes qui y croient?

Nous avons essayé de montrer avec le modèle néoplatonicien que la transformation rituelle de l'objet n'était précisément pas une question de croyance. L'intuition théologique fondamentale du néoplatonisme, c'est que Dieu est, selon une formule bien connue, «au-delà de l'être» (epekeina tês ousias) ${ }^{21}$. Cet axiome théologique a pour conséquence de situer le rapport au divin au-delà de toute appréhension subjective: la divinité est indicible, inconnaissable, incompréhensible. C'est pourquoi le rapport rituel au divin ne saurait se réduire à une forme de connaissance ou de croyance. 
Et ce n'est pas non plus la pensée qui unit les théurges aux dieux. Sinon, qu'est-ce qui empêcherait ceux qui pratiquent la philosophie contemplative d'obtenir l'union théurgique avec les dieux? En réalité, la vérité n'est pas ainsi : c'est l'accomplissement des actes ineffables mis en œuvre au-delà de toute intellection comme il convient au divin et la puissance des symboles indicibles pensés seulement par les dieux, qui établissent l'union théurgique. C'est pourquoi ce n'est pas en utilisant l'intellect que nous accomplissons ces œuvres. Car ainsi, leur mise en œuvre serait intellectuelle et dépendrait de nous. Aucune de ces deux propositions n'est vraie. En effet, sans que nous n'utilisions notre intellect, ce sont les symboles eux-mêmes qui accomplissent à partir d'eux-mêmes leur œuvre propre, et la puissance ineffable des dieux vers lesquels ils remontent reconnaît par elle-même ses propres images, sans être éveillée par notre intellection ${ }^{22}$.

L'intellect est mis hors-circuit dans l'effectuation des rites théurgiques. Ceux-ci ne nécessitent aucune composante subjective pour fonctionner: ce sont les choses elles-mêmes qui agissent, le divin qui se reconnaît dans ses symboles. C'est sur la base de cet argument que les derniers néoplatoniciens ont promu la théurgie au-delà de la contemplation philosophique elle-même, faisant une part plus belle à la pratique qu'à la théorie. Autrement dit, il n'y a pas besoin de croire pour que cela marche. Jamblique formule l'une des premières théories de ce que l'on appellera plus tard l'efficacité symbolique $^{23}$.

C'est précisément là qu' apparaît la seconde difficulté. Pour mettre hors circuit la dimension subjective du rite, on le fait intégralement passer du côté des objets, en considérant le rituel comme une modalité de l'existence même des choses. N'est-ce pas présupposer ce que l'on cherche à démontrer?

Encore une fois, l'idée de la transcendance absolue du divin interdit toute interprétation vulgaire des pratiques télestiques. Il faut reformuler la question: comment une statue peut-elle être divine? de la façon suivante: comment ce qui est absolument transcendant peut-il se retrouver dans les choses? C'est à ce problème épineux que la théorie des signatures apporte une solution géniale. La

22. Jamblique, Les mystères d'Égypte, p. 71-72.

23. Sur la notion d'efficacité symbolique, nous renvoyons à l'étude classique de Lévi-Strauss, «L'efficacité symbolique», in Anthropologie structurale, Paris, Plon, 1958, p. 213-234 (initialement paru dans la Revue de l'histoire des religions, t. $135, \mathrm{n}^{\circ} 1,1949$, p. 5-27), qui souligne la dimension «inconsciente » de l'efficacité rituelle et rapproche par ce biais l'activité chamanique de la pratique psychanalytique. 
signature d'une chose est précisément ce qui la soustrait à ses déterminations naturelles pour la restituer à sa dimension divine. En tant que science des signatures, la théurgie manifeste la présence des dieux sur un mode non pas substantiel mais purement rituel et opératif. C'est symboliquement qu'une statue est un dieu: ce qui ne signifie pas de façon irréelle, mais de façon inessentielle. En ce sens, les pratiques théurgiques ne sont résolument pas une excroissance pathologique du dernier néoplatonisme mais témoignent au contraire de sa cohérence profonde.

La plus grande difficulté tient sans doute au changement de paradigme métaphysique qu'implique l'adoption d'une telle théorie. Penser l'objet rituel - au sens de cet objet paradoxal qui est autre chose que ce qu'il est - suppose deux choses. D'une part de penser l'objet non pas selon ses déterminations essentielles ou matérielles mais selon ses rapports dynamiques. D'autre part de penser la corrélation entre une activité rituelle et le type de qualités qu'elle confère aux objets qu'elle englobe. Le coup de génie de Proclus est de parvenir à articuler ces deux perspectives ensemble, en envisageant le rituel comme la vie secrète des choses mêmes.

En appelant «fétichisme» la croyance illusoire des Africains et des «primitifs» dans le caractère divin, vivant ou animé de certains objets, la culture occidentale moderne a tenté de contourner un problème qui n'a cessé de la hanter et qu'elle est incapable de résoudre parce qu'elle s'est privée des moyens de le poser correctement $^{24}$. L'idée qu'un objet puisse être animé ne nous est plus familière et nous ne pouvons que l'envisager comme une croyance superstitieuse. C'est pourquoi l'anthropologie moderne ne peut que se heurter à des questions insolubles concernant le rapport de la croyance et de l'efficacité des rites ${ }^{25}$. Là où toutefois la religion ne relève pas d'une pure expérience subjective, d'un

24. La notion de «fétichisme» a été introduite par Charles de Brosses en 1760 dans le célèbre essai $D u$ culte des dieux fétiches et elle a connu une immense fortune dans les sciences humaines jusqu'à aujourd'hui. Sur l'histoire de la notion, on lira William Pietz, Le fétiche: généalogie d'un problème, trad. Aude Pivin, Paris, Kargo \& l'Éclat, 2005.

25. Que ce soit dans l'associationnisme d'idées chez Tylor et Frazer, dans la mentalité primitive chez Lévy-Bruhl, dans le fait social chez Durkheim ou Mauss, dans la fonction symbolique chez Lévi-Strauss, la question de l'efficacité nous semble toujours être réduite à celle de la croyance, plus ou moins consciente, en cette efficacité, c'est-à-dire rapportée à une dimension strictement humaine, qu'elle soit psychologique, sociale ou structurale. 
rapport de l'esprit à lui-même, mais se déploie dans un monde de choses, se fait commerce avec des objets, le concept de croyance n'a à peu près aucune valeur heuristique ${ }^{26}$.

Si l'on fait disparaître la difficulté, ce n'est pas en évitant le problème, mais en levant les obstacles à sa bonne formulation: plutôt que de chercher à comprendre comment un rituel peut transformer un objet en une divinité, on cherche à comprendre ce qu'est un objet pour qu'il puisse cristalliser une relation rituelle au divin. C'est ce qu'ont fait les néoplatoniciens au moment historique précis où leur univers spirituel menaçait de s'effondrer en même temps que l'Empire. Nous pensons qu'ils se sont très bien compris eux-mêmes, mieux sans doute que nous ne les comprenons. Ils ne croyaient pas que les statues étaient des dieux, au contraire, ils savaient très bien que les dieux étaient très loin, mais ils savaient aussi comment les faire revenir avec un peu d'herbe et un peu de pierre $^{27}$.

g.casas@free.fr

26. Notons, pour nuancer notre affirmation, que le concept de croyance (pistis) n'est pas absent des théologies chaldaïques et des théories néoplatoniciennes. Il convient toutefois de préciser les choses en soulignant d'une part, que la pistis ne correspond pas à notre idée moderne de croyance, mais consiste en une puissance divine plus qu'en une disposition subjective, et d'autre part, que les néoplatoniciens eux-mêmes opposent une idée supra-rationnelle de la foi comme union et contact avec le divin à la simple croyance comme forme inférieure, voire irrationnelle, de connaissance. Quand Proclus lie explicitement la pistis à la puissance théurgique, ce n'est pas tant pour rapporter l'efficacité rituelle à une faculté subjective que pour signaler son caractère supra-rationnel et transcendant (voir Théologie platonicienne, I, 25, éd. et trad. Henri-Dominique Saffrey et Leendert Gerrit Westerink, Paris, Les Belles Lettres, 1968, p. 109-113). Sur l'élaboration de la notion de croyance dans le néoplatonisme, voir Philippe Hoffmann, «La triade chaldaïque érôs, alètheia, pistis de Proclus à Simplicius», in A.-Ph. Segonds - C. Steel (éd.), Proclus et la Théologie platonicienne. Actes du Colloque International de Louvain (13-16 mai 1998) en l'honneur de H.D. Saffrey et L.G. Westerink $\dagger$, Leuven-Paris, 2000, p. 459-489; Id., «Erôs, Alètheia, Pistis... et Elpis: tétrade chaldaïque, triade néoplatonicienne (fr. 46 des Places, p. 26 Kroll)», in H. Seng - M. Tardieu (ed.), Die Chaldaeischen Orakel: Kontext - Interpretation - Rezeption, Heidelberg 2010, p. 255-324.

27. J'adresse mes remerciements, pour ses précieuses remarques de lecture, à M. Constantin Macris (Centre national de la recherche scientifique/Laboratoire d'études sur les Monothéismes - Centre d'études des religions du Livre). 